\title{
Conformational Molecular Switches for Post-CMOS Nanoelectronics
}

\author{
Alain E. Kaloyeros, Mircea R. Stan, Jr., Senior Member, IEEE, Barry Arkles, Robert Geer, Eric T. Eisenbraun, \\ James E. Raynolds, Anand Gadre, Yongqiang (Alex) Xue, and James Ryan
}

\begin{abstract}
Theoretical treatments forecast that bistable CMOS devices using electronic charge as a state variable will operate at their maximum thermal dissipation limit possibly as early as 2012. The problem is further compounded by increasing manufacturing challenges associated with the ever decreasing logic switch dimensions. These challenges require the development of new fabrication strategies that replace or complement current top-down lithography with bottom-up protocols using controlled self-assembly of nanomaterial building blocks. To answer some of these issues, this paper focuses on a new device paradigm consisting of an arene-metal-arene conformational switch, addressable through capacitive, inductive, or resonant-tunneling field coupling. The operating principle is based on voltage-tunable modulation in quantum electron transmission. The switch is open (OFF) when the metal ion is displaced to a position at a $\mathbf{C}-\mathbf{H}$ bond on the arene ring due to an externally applied bias. Conversely, when the external bias is removed, the metal ion moves to an axis- symmetric position on the arene ring, and the switch is closed $(\mathrm{ON})$. The paper presents a summary of the architecture, operating principle, and advantages of the conformational switch, along with associated findings from proof-of-concept theoretical and experimental studies of its target specifications and performance. The paper also discusses opportunities and challenges related to the integration of conformational switches into hybrid CMOS-molecular and monolithic (all molecular) circuits.
\end{abstract}

Index Terms-Arene-metal-arene (AMA) ring, conformational molecular switch, controlled self-assembly, molecular insertion, silicon nanowires.

\section{INTRODUCTION: LIMITATIONS OF CONVENTIONAL CMOS}

C ONTINUING performance improvements in CMOS integrated circuits (IC) through reduction or "scaling" in device feature size are projected to be severely limited by fundamental physics considerations [1]. One of the most critical of these considerations is passive leakage in the form of quantum mechanical tunneling of electrons across the gate dielectric

Manuscript received February 6, 2007. This work was supported by the NanoElectronics Research Corporation (NERC), through the Institute for Nanoelectronics Discovery and EXploration (INDEX), by the Microelectronics Advanced Research Corporation (MARCO), through the Interconnect Focus Center (IFC), and by the New York State Office of Science, Technology, and Academic Research (NYSTAR). This paper was recommended by Guest Editor C. Lau.

A. E. Kaloyeros, R. Geer, E. T. Eisenbraun, J. E. Raynolds, A. Gadre, Y. Xue, and J. Ryan are with the College of Nanoscale Science and Engineering (CNSE), University at Albany-State University of New York (SUNY), Albany NY 12203 USA (e-mail: akaloyeros@uamail.albany.edu).

M. R. Stan is with Electrical and Computer Engineering Department, University of Virginia, Charlottesville, VA 22904 USA.

B. Arkles is with Gelest Inc., Tullytown, PA 19007 USA.

Digital Object Identifier 10.1109/TCSI.2007.907827

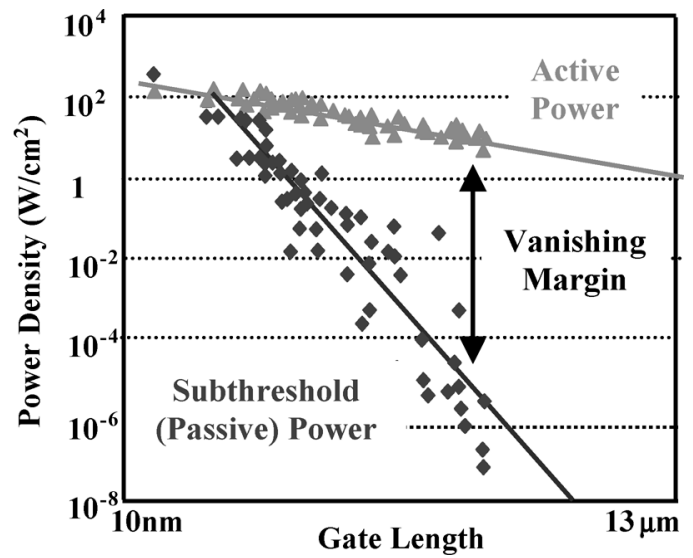

Fig. 1. Active and leakage power density trends versus FET gate length (adapted from [2]).

layer to the field-effect transistor (FET) channel region (gate leakage) [2] as well as the subthreshold leakage current, which both increase exponentially with scaling at a higher rate than the active current. This trend is illustrated in Fig. 1 which plots overall chip power density against FET gate length. The increase in leakage power density with decreasing feature size, and the need to perform irreversible logic operations with energy dissipation $>k_{B} T$, are projected to halt CMOS scaling as the key enabler of the historical rate of progress of semiconductor technology.

At the same time, the basic physics of electron scattering is quickly becoming a major limiter to the performance of conventional electrical interconnects in CMOS ICs [3], [20]. Fig. 2 illustrates the effect of electron scattering in $\mathrm{Cu}$ wires, as the wire width $D$ is reduced below the mean free path for electron scattering $\lambda_{\mathrm{e}}(39 \mathrm{~nm}$ in $\mathrm{Cu})$. Although scattering of electrons from morphological irregularities (grain boundaries, interfacial roughness) of $\mathrm{Cu}$ lines can be minimized (even perhaps eliminated) with improved structural control during interconnect processing, physics provides no known solution to electron scattering from even a structurally ideal $\mathrm{Cu}$ interface/surface. These issues are exacerbated by limitations in lithography and materials processing that dramatically increase feature-to-feature and device-to-device variability. Examples include the rise in line-edge roughness (LER) in lithographic pattern transfer with decreasing feature size, and the variability associated with atomic-layer uniformity in ultrathin and "zero-thickness" films [4]. Nonoptimal LER can lead to variations in local interconnect resistivity or compromise interconnect diffusion barrier layers and threaten IC reliability. Similarly, thickness nonuniformity 


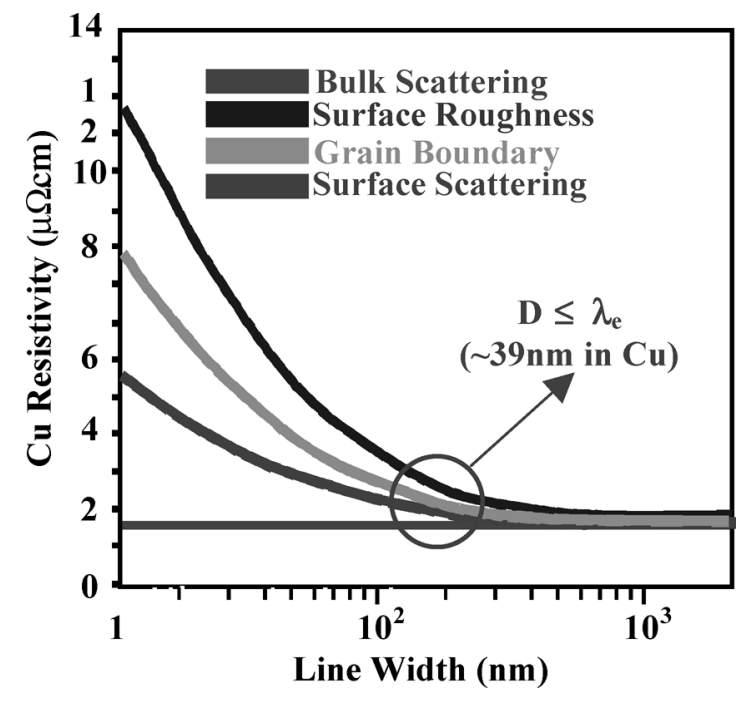

Fig. 2. Cu line resistivity versus line width (adapted from [3], [20]).

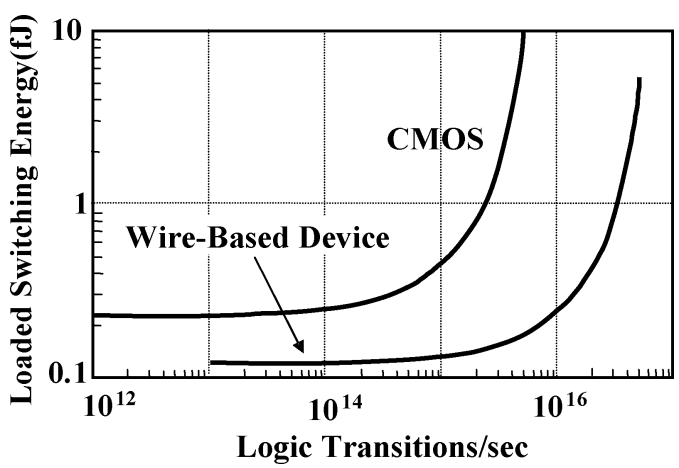

Fig. 3. Comparative plots: (a) actual performance of current CMOS (red curve, adapted from [7]), and (b) qualitative potential enhanced performance for lowcapacitance, high-density wire-like devices (green curve).

on the order of a single atomic layer in FET gate dielectric can increase the leakage current by an order of magnitude.

\section{EXTENDING CMOS: SELF-ASSEMBLED WIRE-LiKE FETS}

A partial "work-around" solution for these limitations is to exploit atomic and molecular self-assembly to form quasi-1D FETs, most notably carbon nanotubes (CNTs) and Si nanowires (SNWs). Although the operational principles are essentially unchanged from that of conventional CMOS, the wire-like geometry of CNTs and SNWs offer possible advantages in device performance, capacitance, and device pitch [5]-[7]. Fig. 3 plots loaded switching energy versus total logic transitions per section for conventional CMOS compared to a possible wire-like FET-based logic. The advantage of wire-like FETs presumes that appropriate processing and fabrication paradigms can be developed to reach a device pitch $\sim 10$-nm with corresponding solutions for channel doping, contact formation, and interconnect density. The complexity of the latter, however, is seen as a potential advantage since CNTs or SNWs can, in principle, be utilized for both device and interconnect functionality, obviating the need for dissimilar interconnect materials, at least at the local wiring level, and eliminating the challenges associated with hybrid integration of radically different material sets.
Still, the fundamental electrostatics of FET operation are not inherently altered by a CNT- or SNW-based platform and are subject to the same switching-energy constraints $\left((1 / 2) \mathrm{CV}^{2} \gg k_{B} T\right)$ and gate dielectric limitations of conventional Si FETs. Consequently, there continues to be a need for an alternate switching paradigm which can overcome the limitations of the FET's charge-based state variable.

\section{CONFORMATIONAL SWITCHING IN QUASI-1D MOLECULAR WIRES}

As noted by one of the authors (Xue) in prior work, the current challenges facing conventional FET paradigms may necessitate the exploitation of electron and electronic charge/current in fundamentally new ways that are closely linked to the use of alternate state variables for information transfer [8], [21]-[23]. The same conclusion was reported recently by Bourianoff [9]. One such approach is described herein for the fabrication of an alternate switching element based on electrostatically-mediated molecular conformational modulation on a 1D, Si-based molecular quantum channel conductor. This approach builds on the wire-like device/interconnect paradigm discussed above in order to enable a pathway to homogeneous or monolithic integration, while maintaining a viable route to a sub-10-nm device pitch.

The fundamentals of conformational switching in 1-D stacked arene systems builds from recent work by Avouris and coworkers [10]. Their theoretical study of the formation of 1 -D quantum channels focuses on the influence of $\pi$-orbital coupling in a 1-D stack of 4,4'-biphenyldithiol (BDT) and the resulting electronic and electrical properties of the assembly as the intermolecular distances are reduced below $0.4 \mathrm{~nm}$. In the regime of strong $\pi$-interactions the system showed large conductance modulations upon application of a transverse (gate) field. The switching mechanism involves a delocalized $\pi$-resonance, i.e., resonant tunneling in the intermolecular $\pi$ - and $\pi^{*}$-bands of the BDT stack. They found the quantum channel transmission to be significantly higher in the $\pi^{*}$-resonance configuration.

One of the authors (Raynolds) participated in extending this work through the use of local-orbital-basis density-functional theory and Green's-function-based simulations to study the $I-V$ characteristics, applied voltage effects, and band alignments of stacked arene systems.

Their simulations showed that controlling the chemical structure of the $\pi$-conjugated arene elements was essential to optimizing 1-D quantum-channel transport. More specifically, geometry-confined phenolate/benzene dia- zonium pairs between $\mathrm{Au}$ (111) contacts exhibited a substantial conductivity increase at low bias voltages, as compared to benzene-based molecular wires [11]. More recently, Raynolds and coworkers evaluated the phenolate/benzene system in terms of finite-temperature behavior and conformational stability using a similar theoretical simulation approach. This work examined a Peierls distortion stemming from electron-phonon interactions. A mechanical metal-insulator transition was observed [Fig. 4(a) and (b)] whereby conformational dimer formation between adjacent phenolate-benzenediazonium pairs formed a 2-eV bandgap [12]. This result and the earlier work of Avouris [10] 


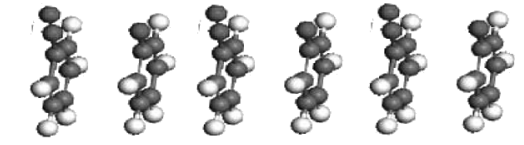

Band Structure of 1D Stacked Pairs

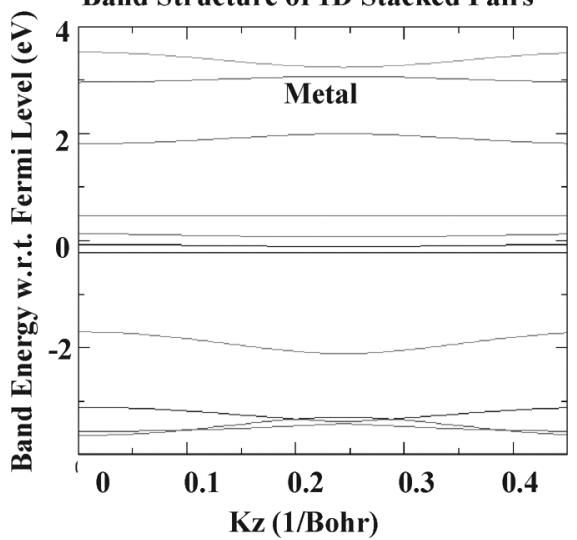

(a)

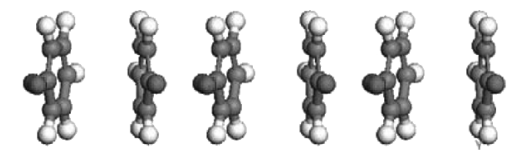

Band Structure of 1D Stacked Dimers

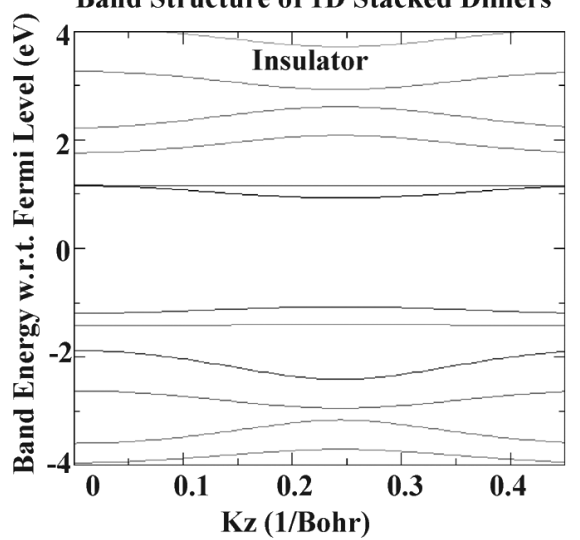

(b)

Fig. 4. (a) Energy diagram for a 1-D phenolate/benzenediazonium quantum channel without Peierls distortion. (b) Energy diagram for 1-D phenolate/benzenediazonium quantum channel in the presence of a Peierls distortion.

and Liu [11] open a pathway for a molecular conformational route to quantum channel switching through manipulation of arene conformation in a quantum molecular conductor.

\section{Monolithic Device/InTERConnect Concept BASED on CONFORMATIONAL MOLECULAR SWITCHING}

Work by the authors under the NanoElectronics Research Corporation (NERC) Institute for Nanoelectronics Discovery and EXploration (INDEX) has focused on a multi-phase program to develop and deploy a new device paradigm that transcends electronic charge as the state variable. The device consists of a metal-arene based conformational switch, addressable through capacitive, inductive, or resonant-tunneling field coupling, as shown schematically in Fig. 5.

When biased, the quantum conductance of this arene-metal-arene (AMA) switch is controlled by molecular

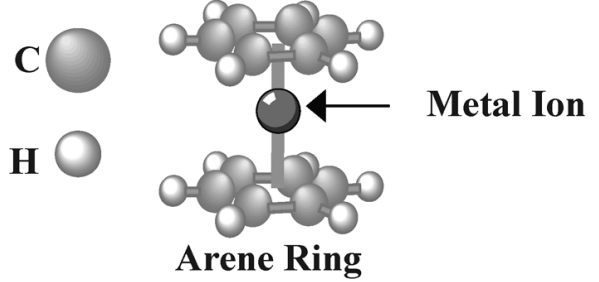

Fig. 5. Quantum conductance of a biased AMA switch is controlled by molecular conformation, i.e., the relative metal ion position.

conformation, i.e., the relative metal ion position. Fig. 6 displays the operation of the molecular switch as compared to that of a conventional n-FET transistor. As shown in Fig. 6(a), the application of a bias voltage $V_{g}$ induces a molecular conformation change through a displacement of the metal ion from the $\pi$ bond configuration, thus yielding an OFF (0) state. The corresponding state for a conventional n-FET transistor is also displayed for comparison purposes. Conversely, in the absence of a bias voltage $V_{g}$, the metal ion aligns in the $\pi$ bond configuration, thus yielding an ON (1) state, as shown in Fig. 6(b). The corresponding ON state for a conventional n-FET is also displayed.

The AMA switch has a number of attractive features, including its functional scalability and ability to operate within simple integrated circuitry. It holds the promise for monolithic (fully molecular) computer chip architectures given that the same molecular building blocks (same state variable) can be used to develop the molecular interconnect/wiring platform (e.g., SNW) and the molecular switch [3], [20]. In one such embodiment, a field-switchable conformational transition in the molecular device could modulate the quantum-channel transmission in the SNW, which would conduct through a ballistic electron transport mechanism. This feature is highly desirable since it can potentially eliminate the need for hybrid integration and associated material, process, and performance challenges.

Furthermore, the molecular switch can be fabricated using processes that are fully compatible with, and integratable into, current semiconductor process flows. In particular, modifications to current electroplating methodologies, such as molecular plating, have been shown to achieve controlled self-assembly of molecular wires on standard Si wafer templates [3], [20].

Prior work by the authors has demonstrated the efficacy of self-assembling carbon-based molecular backbones to position arene-based pendant groups [2], [14], [15]. However, charge transfer across molecular contacts in these systems was shown to be the overall performance limiter. In contrast, the utilization of a Si-based molecular conductor as interconnect ensures reduced contact resistance through the concept of molecular insertion (this is discussed in more detail in Section V).

Modeling studies for quantum channel transition in a metal-arene system incorporating a metal ion- $\pi$ conjugated arene pair, namely benzene-lithium-benzene, are shown in Fig. 7 [13]. An externally applied bias induces a displacement of the $\mathrm{Li}$ ion from the ON (1) state, where the $\mathrm{Li}$ ion is located at an axis-symmetric position on the benzene ring, to the OFF (0) state, where the $\mathrm{Li}$ ion is located at a $\mathrm{C}-\mathrm{H}$ bond. 


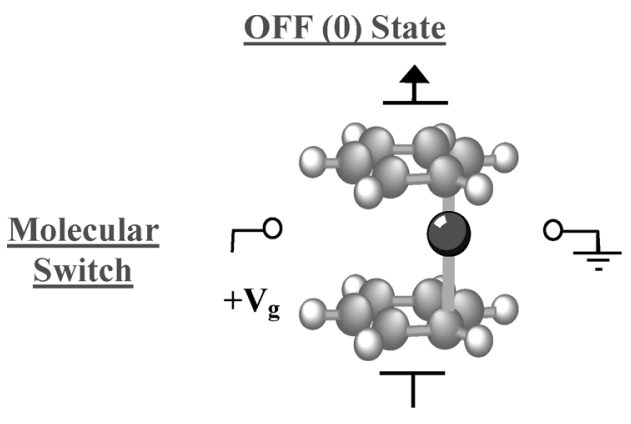

Metal Ion Displaced from $\pi$-bond Via biased voltage $\left(V_{g}\right)$ Induced Conformation Change

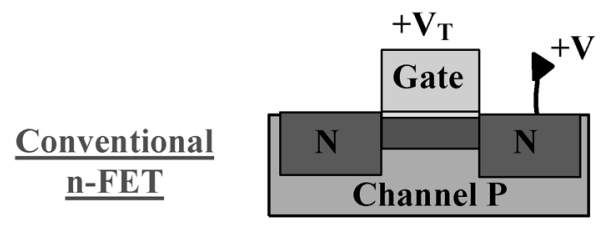

Channel Depleted $\left(\mathbf{V}_{\mathrm{g}}<\mathrm{V}_{\mathrm{T}}\right)$

(a)

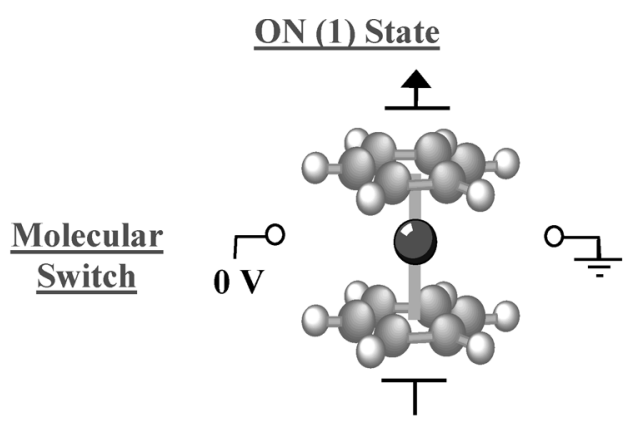

Metal Ion Aligned with $\pi$-bond

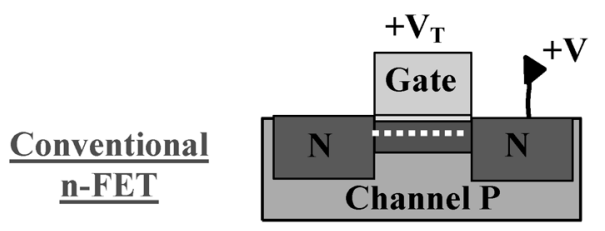

Charge Inversion. Channel Conducts $\left(V_{g}>V_{T}\right)$

(b)

Fig. 6 (a) Application of a bias voltage to the molecular switch induces a molecular conformation change through a metal ion displacement from the $\pi$ bond configuration, thus yielding an OFF (0) state. (b) In the absence of a bias voltage, the metal ion in the molecular switch aligns in the $\pi$ bond configuration, thus yielding an ON (1) state.

The switch operates therefore on the principle of voltagetunable modulation in quantum electron transmission. In this case, the conformational energy difference between the ON and OFF states can be engineered to be $\sim 0.25 \mathrm{eV}$. The resonant (maximum) conformational frequency for a single metal ion could be as high as $\hbar /(0.25 \mathrm{eV}) \sim 10^{14} \mathrm{~Hz}$, although a performance trade-off exists between terahertz device switching speeds and conformational stability. The conformational stability of the displaced metal ion depends upon boundary conditions, as determined by the polarizability of the local chemical (a) Li Ion at Center of Ring
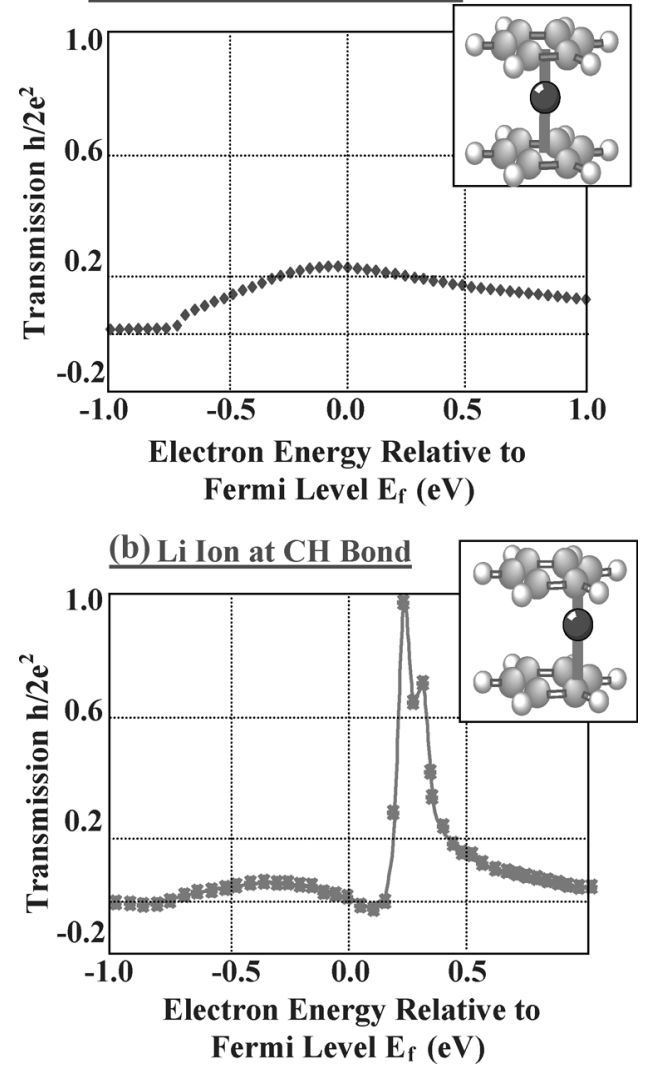

Fig. 7. In the absence of external bias, the Li ion is displaced to an axis-symmetric position on the benzene ring (a), from a bias-induced position at a $\mathrm{C}-\mathrm{H}$ bond (b). This displacement results in a voltage-tunable modulation in electron transmission and provides a pathway for a conformational switch.

bonding environment, as well as on the presence of "gating" and "source-drain" electric fields.

\section{MONOLITHIC DEVICE/INTERCONNECT FABRICATION STRATEGIES}

The AMA switch can be fabricated using tightly controlled and precisely engineered fabrication protocols that are compatible with current silicon semiconductor fabrication flows. Prior work in this area has focused on metallization of molecular systems through a solution-based attachment of metal ion particles onto complex biological structures such as DNA, viruses, or bacteria. The latter is achieved pre- or post-translationally, i.e., either before or after the molecular system is assembled at, or transferred to, the substrate [16], [17].

The fabrication process developed by the authors builds on these prior studies but involves instead the use of inorganic molecular structures, namely polysilanes, owing to their enhanced thermal stability, simplified synthetic characteristics, and their ability to form uniform, sterically defined, molecular lengths and configurations. Furthermore, while the central silane chain is intrinsically conductive via delocalized $\pi$ bonding, the resulting well-defined structure can also be employed as a building block suitable for controlled nanoscale self-assembly and flexible charge transport motifs. A case in point is $\pi$ bond conjugation of neighboring phenylic groups or metallic conduction via ordered nanoparticles. 


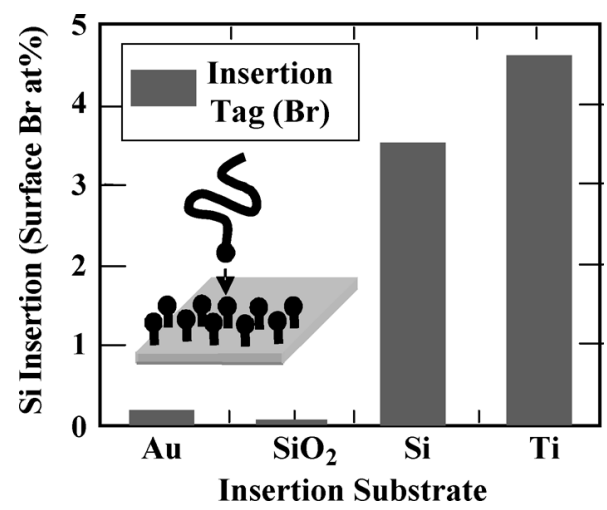

Fig. 8. Surface affinity for bromine-tagged silane attachment as function of substrate type. The affinity is represented by surface atomic concentration of $\mathrm{Br}$ as determined via XPS. Inset schematic drawing references Br-tagged silane to a Si surface. The ratio of $\mathrm{Si}$ to $\mathrm{SiO}_{2}$ selectivity $(\sim 70: 1)$ is consistent with the observation of molecular insertion reported in the literature [18].

A critical and motivating factor for the use of a Si-based quantum-channel platform for a metal-arene conformational molecular switch is its inherent ability for integration onto $\mathrm{Si}$ semiconductor structures. This feature offers the potential to form intrinsically high-performance, low-impedance molecular contacts to a metal or semiconducting substrate. As such, this characteristic further differentiates the Si-based nanowire approach from its carbon-based analogs, such as CNTs or graphene, where the realization of good electrical contacts to conventional metal and semiconductor leads and structures remains a challenge.

Prior experimental studies, by the authors, of controlled "molecular insertion" in SNW quantum channels have focused on the incorporation of elementally tagged $(\mathrm{Br})$ polysilane molecules into a self-assembled structure on prevailing semiconductor, metal, and insulator substrates to differentiate covalent attachment. Results are shown in Fig. 8, which plots surface $\mathrm{Br}$ atomic concentration in the self-assembled structures, as determined by x-ray photoelectron spectroscopy. The data supports the existence of a strong substrate surface effect on molecular adhesion strength. In particular, the ratio of surface attachment selectivity of Br-tagged silane between $\mathrm{Si}$ and $\mathrm{SiO}_{2}(\sim 70: 1)$ is consistent with the observation of controlled molecular insertion, as reported previously in the literature [18]. This demonstration is a critical enabling step for the establishment of electrically viable molecular-level contact points between the conformational switch structure and conventional semiconductor structures.

In addition to contact formation, a critical aspect of the development of reliable molecular devices is the identification of fabrication schemes that can provide atomic scale control and reproducibility in the growth and stability of the resulting structures. In this context, the rigidity of the polysilane chain is modulated by steric effects of the selected substituent chemistry, and appropriate design of the polysilane molecule can enable a length longer than $>100 \mathrm{~nm}$, while minimizing intermolecular entanglement [19]. These features lead to the possibility of using regularly-spaced functional groups as initiation points for the selective formation or attachment of metal and organic groups, particularly the interleaved metal-arene building blocks

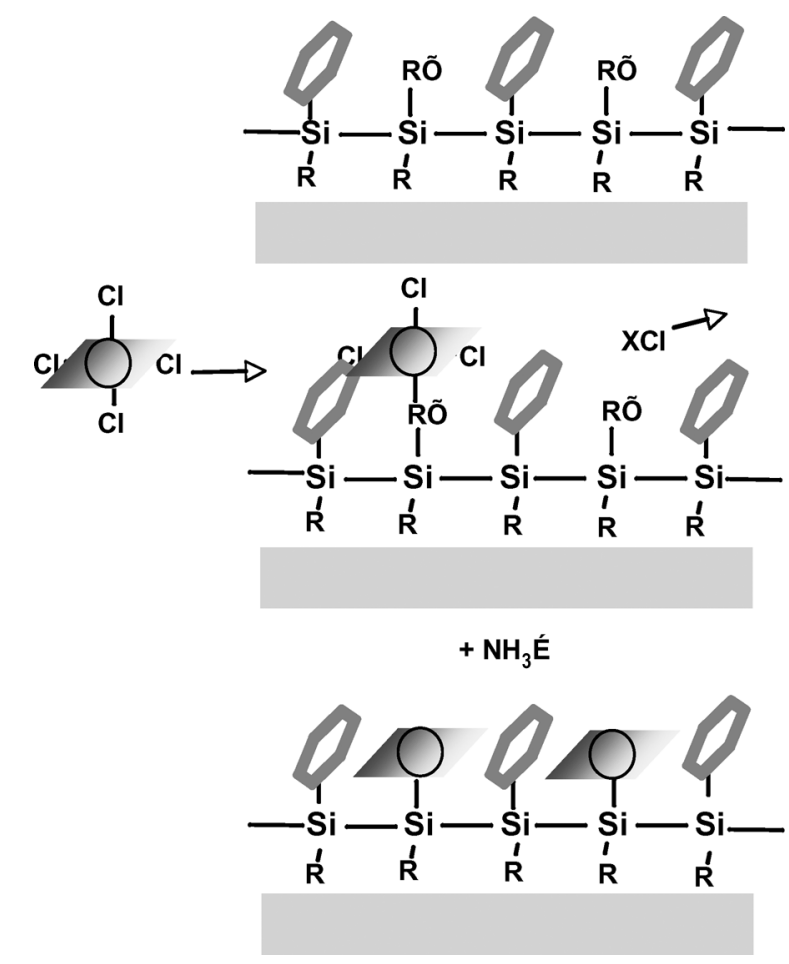

Fig. 9. The "Molecular ALD" fabrication scheme, wherein polysilane functional groups $\left(R^{\prime}\right)$ are selected to function as reactive exchange group for the ALD surface reactions necessary for the fabrication of the conformational switch structure.

used in the formation of the conformational switch. For this purpose, work by the authors has focused on the development of atomic layer deposition (ALD) or molecular plating schemes that utilize a polysilane molecule synthesized with an appropriate series of side groups amenable to subsequent ALD or molecular plating type surface growth processes. In particular, by exploiting common polysilane functional groups including halides, hydroxyls, and alkyls that are known to act as exchange groups for ALD reactions or selective solution-phase plating reactions, standard and well-characterized arene and metallization chemistries can be integrated in the fabrication of the molecular device and interconnect schemes desired. This approach is shown schematically in Fig. 9. For illustration purposes, findings from a proof-of-concept investigation of controlled self assembly using selective solution-based plating are shown in Fig. 10.

\section{Integrated Molecular Switch Design: Circuits AND DESIGN STRATEGY}

Ongoing research by the authors has focused on the design and fabrication of a circuit design methodology that provides a way to implement simple combinational (gates) and sequential (registers) logic circuits $[20,21]$. In this approach, memory can be implemented as a collection of registers, or, ideally, using specialized structures that take advantage of the special regularities associated with memory design.

Logic design using molecular switches is straightforward and consists of networks of series and parallel switches that lead to direct implementation of AND and OR (for pass-transistor style networks, see Fig. 11), or of NAND, NOR, and INV (for restoring 


\section{Directed Molecular Nanowire Self-Assembly}

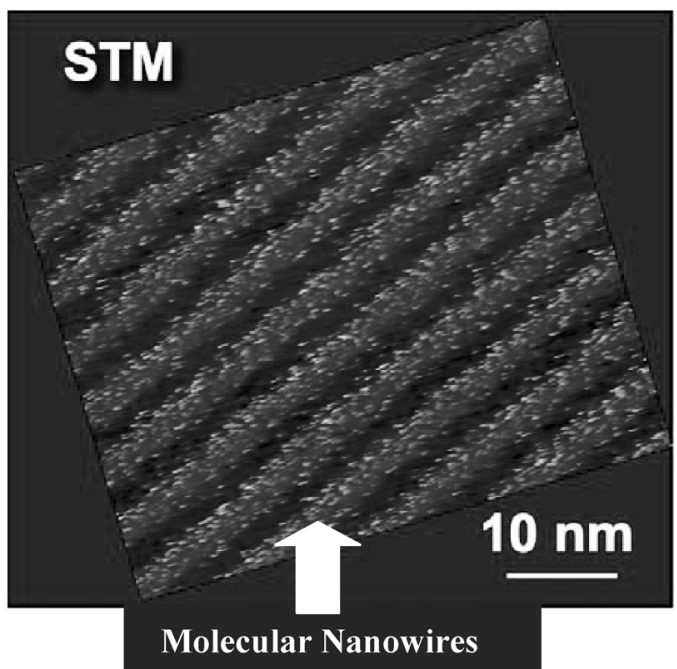

Fig. 10. Controlled self assembly of molecular nanowires using selective solution-based plating.

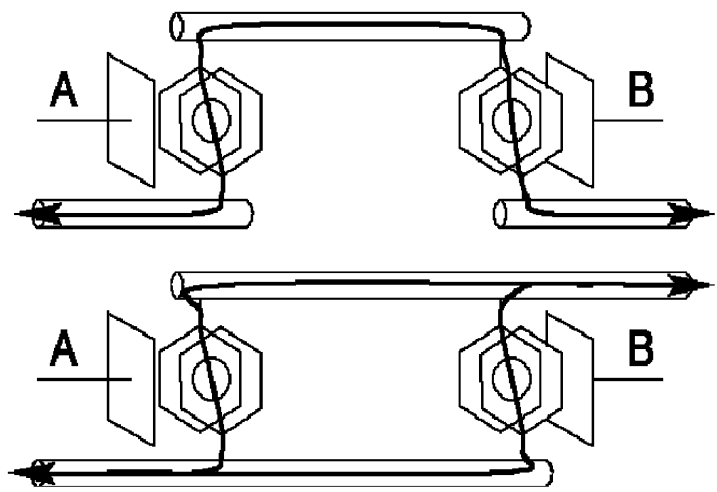

Fig. 11. Series and parallel switch structures for logic function implementation: top-series connection, ON when both A and B are ON, bottom-parallel connection, ON when A or B are ON.

gates with pull-up and pull-down networks). More complex logic functions [sum-of-product (SOP), or product-of-sums (POS)] functions can also be directly implemented with more complex networks, or complex circuit functionality can be obtained in a hierarchical way by using simple "universal gates" such as NANDs and NORs.

Memory design can use two sets of orthogonal nanowires, known as rows and columns, with a nonvolatile switch at the intersection of each set of two orthogonal wires (see Fig. 12). A voltage applied on one of the nanowires can be sensed as a signal on the orthogonal nanowire if the switch between two such nanowires is ON, but not when the switch is OFF. There are several variations on how the signal is sensed (e.g., using voltage or current sensing, single-ended or differential, etc.) as well as to how the voltages are applied, and to how the inactive nanowires are connected to various reference voltages [22]. The nonideal behavior of the switches and the existence of "sneak current" paths in the circuit (basically, even switches that are OFF still have a finite resistance that affects the signal to noise

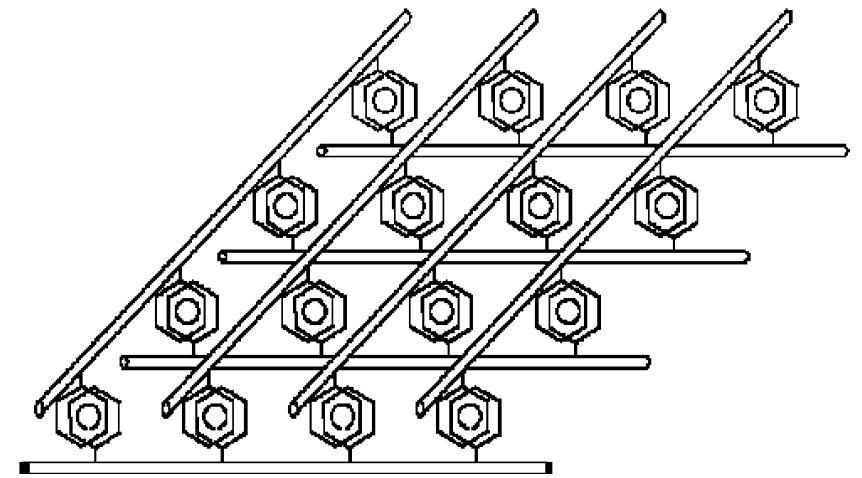

Fig. 12. Crossbar memory structure with arene-Me-arene stacks at each intersection of orthogonal SNWs.

ratio of the system) makes the detection of the state of the switch nontrivial for large arrays.

Using "directional" switches with a diode-like characteristic which let current pass in a single direction is a critical capability that avoids most of the problems with sneak currents in crossbars. Accordingly, the authors are actively investigating methods to obtain stacked-arene type devices with unidirectional properties to be used in crossbar memory structures. Still, small usable memory structures can be obtained even with regular bidirectional switches.

In this respect, high level evaluation of circuit behavior can be made based on ideal switch characteristics. Detailed and accurate estimation of performance, noise immunity, power, can only be done by using Spice-like simulations. Molecular device behavior is very complex and hard to capture in simple compact models as required by Spice, but at this point there are no better alternatives. Spice compact models can be "physical" if they are described by equations derived from the actual physical phenomena, or "empirical" if the equations are derived for a "best fit" without an emphasis on the actual physical behavior. Physical models are preferable as they are more likely to scale and be able to extrapolate, although they are also much harder to derive for various types of devices. In this respect, one of the authors (Stan) has developed an empirical compact modeling methodology captures the salient physical characteristics of the device [23]. Such compact models can be derived either from experimental data (measurements) or from ab-initio calculations as described in Sections III and IV. Current studies are using the second path (ab-initio calculations) to derive compact models for circuit design. These models are validated with actual measurements of molecular device structures as they are developed and optimized. Similar models need to be also derived for the interconnect, since the polysilane SNWs and the stacked-arene wires have as complex behavior as actual devices due to their small dimensions.

In summary, the design of circuits based on molecular devices and interconnect needs at a minimum a schematic capture and a simulation tool, but for complex system design a much more comprehensive infrastructure is needed that includes ways to describe higher level functionality (e.g., by the use of hardware description languages), a way to abstract away functionality from structure, logic synthesis and mapping, physical design, etc. In 
the short term, there is also a need for tools that can help with hybrid (CMOS/nano) circuitry co-design. Such a design infrastructure is an absolute requirement, but, despite many efforts, still not available.

\section{CONCLUSION}

Although the development of a viable nonCMOS based solution to act as the next technological driver for the semiconductor industry is still in its infancy, the work reported here demonstrates the potential for the molecular AMA type switch to eventually be such a solution. The switch exhibits a number of attractive features, including its functional scalability and ability to operate within simple integrated circuitry. It holds the promise for monolithic (fully molecular) computer chip architectures given that the same molecular building blocks (same state variable) can be used to develop the molecular interconnect/wiring platform (e.g., SNW) and the molecular switch. In this case, a field-switchable conformational transition in the molecular device would modulate quantum-channel transmission in the SNW, which would conduct through a ballistic electron transport mechanism. This feature is highly desirable since it can potentially eliminate the need for hybrid integration and associated material, process, and performance challenges. Furthermore, the molecular switch could be fabricated using processes that are fully compatible with and integratable into current semiconductor process flows. Theoretical and experimental proof-of-concept work by the authors has provided early indication of the feasibility of critical milestones in the development of the molecular switch, including target performance specifications, controlled self-assembly on conventional $\mathrm{Si}$ structures, and integration within simple computer circuitry. However, significant challenges remain to be resolved before the concept of a conformational molecular switch can be considered a workable option to replace CMOS in future integrated circuits.

\section{REFERENCES}

[1] International Technology Roadmap for Semiconductor, ITRS, Santa Clara, CA, 2005 [Online]. Available: http://public.itrs.net

[2] E. Nowak, IBM J. Res. Dev., vol. 46, pp. 169-180, 2002.

[3] A. E. Kaloyeros, E. T. Eisenbraun, J. Welch, and R. E. Geer, Semiconductor Int., vol. 26, pp. 56-63, 2003.

[4] B. J. Lin, Microelectron. Eng., vol. 83, pp. 604-613, 2006.

[5] J. Appenzeller, Y.-M. Lin, J. Knoch, and P. Avouris, Phys. Rev. Lett., vol. 93, p. 196805, 2004.

[6] Y.-M. Lin, J. Appenzeller, J. Knoch, and P. Avouris, IEEE Trans. Nanotechnol., vol. 4, pp. 481-489, 2005.

[7] D. J. Frank, IBM J. Res. Dev., in press.

[8] Y. Xue and M. A. Ratner, Nanotechnology: Science and Computation, J. Chen, N. Jonoska, and G. Rozenberg, Eds. Berlin: Springer, 2006.

[9] G. Bourianoff, IEEE Computer, vol. 36, no. 8, pp. 44-53, 2003.

[10] A. Rochefort, R. Martel, and P. Avouris, Nano Lett., vol. 2, pp. 877-880, 2002.

[11] X.-Y. Liu, J. E. Raynolds, C. Wells, J. Welch, and T. S. Cale, J. Appl. Phys., vol. 98, pp. 33712-33715, 2005.

[12] X.-Y. Liu, J. E. Raynolds, C. Wells, J. Welch, and T. S. Cale, in Proc. IEEE 2005 Int. Conf. Simulation of Semiconductor Processes Devices, 2005, pp. 271-274.

[13] C. Wells, J. E. Raynolds, and J. Welch, unpublished results.

[14] A. E. Kaloyeros, E. T. Eisenbraun, J. Welch, and R. E. Gee, Semiconductor Int., vol. 28, pp. 46-52, 2005.

[15] N. I. Topilina, S. Higashiya, N. Rana, V. Ermolenkov, C. Kossow, A. Carlsen, S. C. Ngo, C. C. Wells, E. T. Eisenbraun, K. A. Dunn, I. K. Lednev, R. E. Geer, A. E. Kaloyeros, and J. T. Welch, Biomacromolecules, vol. 7, pp. 1104-1111, 2006.
[16] M. Warner and J. E. Hutchison, Nat. Mater., vol. 2, pp. 272-277, 2003.

[17] J. L. Yu, I. A. Banerjee, and H. Matsui, J. Mat. Chem., vol. 14, pp. 739-743, 2004.

[18] T. M. Owens, K. T. Nicholson, M. M. Banaszak Holl, and S. Süzer., $J$. Amer. Chem. Soc., vol. 124, p. 6800, 2002.

[19] A. Ohira, S.-Y. Kim, M. Fujiki, Y. Kawakami, M. Naito, G. Kwak, and A. Saxena, Chem. Commun., vol. 25, p. 2705, 2006.

[20] S. M. Rossnagel and H. Kim, in Proc. 2001 Int. Interconnect Technol. Conf., 2001, vol. 3.

[21] Y. Xue, S. Datta, and M. A. Ratner, Chem. Phys., vol. 281, pp. $151-170,2002$.

[22] Y. Xue and M. A. Ratner, Inter. J. Quantum Chem., vol. 102, pp. 911-924, 2005.

[23] Y. Xue and M. A. Ratner, Phys. Rev. B, vol. 70, pp. 1554081-155408-6, 2004.

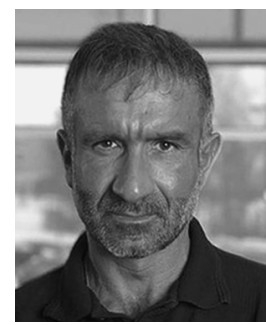

Alain E. Kaloyeros received the Ph.D.degree in experimental condensed matter physics from the University of Illinois, Urbana-Champaign, in 1987.

$\mathrm{He}$ is a Professor of Nanosciences and Vice President and Chief Administrative Officer in the College of Nanoscale Science and Engineering (CNSE), University at Albany (UAlbany)—State University of New York (SUNY), Albany. He has authored and co-authored over 150 articles and contributed to seven books on topics pertaining to nanoelectronics and nanooptoelectronics He has mentored $31 \mathrm{Ph} . \mathrm{D}$. students and over 50 Master's students. He holds 13 U.S. patents.

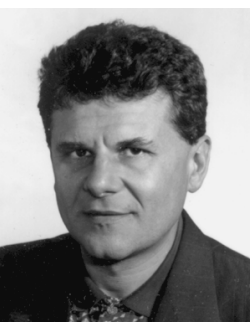

Mircea R. Stan Jr. (SM'98) is an Associate Professor in the Electrical and Computer Engineering Department, University of Virginia, Charlottesville, teaching and researching the areas of high-performance and low-power VLSI, temperature-aware circuits and architecture, embedded systems, and nanoelectronics.

Prof. Stan was a recipient of the National Science Foundation CAREER Award. He was coauthor Best Paper Awards at the Great Lakes Symposium on VLSI (GLSVLSI) 2006, nternational Symposium on Computer Architecture (ISCA) 2003, and Self-Healing, Adaptive and Self-MANaged Systems (SHAMAN) 2002. He was TPC chair of the International Symposium on Low-Power Electronics and Design (ISLPED) 2005 and NanoNets 2007, and general chair of GLSVLSI 2003 and ISLPED 2006. He is a member of the Association for Computing Machinery (ACM), Information and Enterprise Technology (IET), Eta Kappa Nu, Phi Kappa Phi, and Sigma Xi.

Barry Arkles is President and CEO of Gelest Incorporated (a leading chemical supplier of a leading supplier of silanes, silicones and chemical compounds of germanium, tin and lead), Tullytown, PA. His company also supplies metal alkoxides, diketonates and carboxylates.

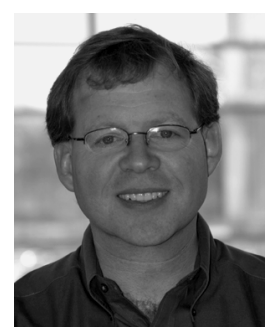

Robert Geer is an Associate Professor of NanoSciences and Assistant Vice President of Academic Affairs in the College of Nanoscale Science and Engineering (CNSE), University at Albany (UAlbany) - State University of New York (SUNY), Albany. His current areas of research include investigations of self-assembling, $\mathrm{Si}$-based molecular wires and devices for next generation integrated circuits, and development of nanoscale metrology tools for measurement and characterization of nanodevice structures including nano-acoustic imaging systems for carbon nanotubes, nanobelts and nanowires, and nano-optical systems for strain metrology in advanced CMOS device structures. He also directs R\&D programs in nanoparticle-based planarization processing for computer-chip manufacturing. 


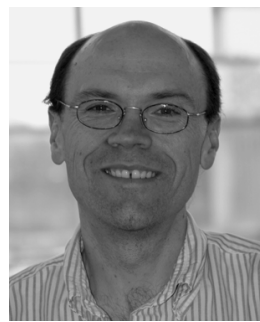

Eric T. Eisenbraun is currently an Assistant Professor in the College of Nanoscale Science and Engineering (CNSE), University at Albany (UAlbany) - State University of New York (SUNY), Albany. Since joining UAlbany in 1998, he has been involved in various areas of nanoscale thin film research for nanoelectronic, thermal, and protective coatings applications. This research includes CVD and ALD of a variety of metallic and dielectric coatings, as well as research involving three-dimensional wafer-scale integration. In addition, he is currently investigating the development of systems based on molecular crystals for applications in nanoscale devices and interconnects. He has over16 years of experience in nanomaterials processing and integration. He has over 50 publications and holds one patent.

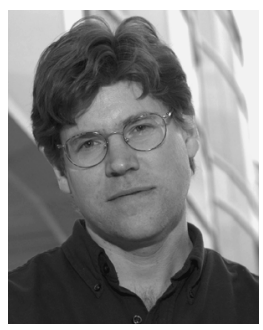

James E. Raynolds is an Assistant Professor of nanoscience in the College of Nanoscale Science and Engineering (CNSE), University at Albany (UAlbany) - State University of New York (SUNY), Albany. His research interests include molecular electronics, spintronics, density functional theory, path integrals, high-performance computing and photonics (antenna's, frequency selective surfaces, and cavity effects).

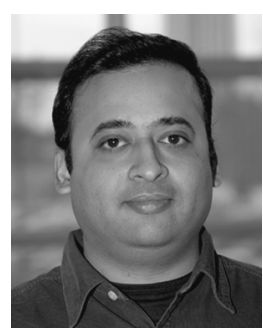

Anand Gadre received the Ph.D. degree in polymer physics from the University Department of Chemical Technology, Mumbai, India, in 2001.

$\mathrm{He}$ is currently an Assistant Professor in nanobioscience in the College of Nanoscale Science and Engineering (CNSE), University at Albany (UAlbany)-State University of New York (SUNY), Albany. His areas of research include microelectromechanical systems (MEMS), bioMEMS, polymeric LED and biofluidic-transdermal microsystems, microsensors, conducting polymers, and

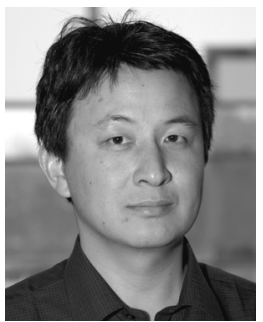

Yongqiang (Alex) Xue received the B.S. degree in applied mathematics from Peking University, Beijing, China, in 1991, the M.S. degree in physics from the University of Massachusetts, Amherst, in 1996, and the Ph.D. degree in solid state devices and materials from Purdue University, Lafayette, IN, in December 2000.

$\mathrm{He}$ is currently an Assistant Professor of nanoscience in the College of Nanoscale Science and Engineering (CNSE), University at Albany (UAlbany) - State University of New York (SUNY), Albany. His research focuses on theoretical and computational study of fundamental nanoscale phenomena and processes in functional nanosystems.

Dr. Xue is a member of Antennas and Propagation Society, ACS, MRS, and OSA.

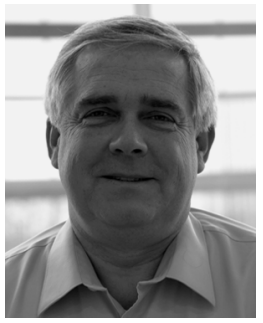

James Ryan is Associate Vice President of Technology and Professor of Nanoscience in the College of Nanoscale Science and Engineering (CNSE), University at Albany (UAlbany) - State University of New York (SUNY), Albany. He joined CNSE in February 2005 after a 25-year career with IBM. From 2003 to 2005, he was a Distinguished Engineer and Director of Advanced Materials and Process Technology Development and served as the site executive for IBM at Albany NanoTech. Prior to that assignment, he managed interconnect technology groups in research, development and manufacturing engineering areas at IBM. $\mathrm{He}$ is the author of over 90 publications and has 47 U.S. patents.

Dr. Ryan is the recipient of numerous awards including 17 IBM invention plateaus, an IBM Corporate Patent Portfolio award, an IBM Division Patent Portfolio Award, IBM Outstanding Technical Achievement Awards for Dual Damascene and for Copper technologies, and the 1999 SRC Mahboob Khan Mentor Award. rechargeable batteries. 